\title{
SQLMutation: A tool to generate mutants of SQL database queries
}

\author{
Javier Tuya, Ma José Suárez-Cabal, Claudio de la Riva \\ University of Oviedo (SPAIN) \\ \{tuya $\mid$ cabal $\mid$ claudio\}@uniovi.es
}

\begin{abstract}
We present a tool to automatically generate mutants of SQL database queries. The SQLMutation tool is available on the Web and it can be accessed using two different interfaces: A Web application to interactively generate the mutants and a Web service that allows it to be integrated with other applications developed using different platforms.
\end{abstract}

Keywords: Database testing, SQL testing, SQL query, Mutation operators

\section{Introduction}

The Structured Query Language (SQL) [6] is a semi-declarative language used by the applications to access the information stored in relational database systems.

The most frequent SQL queries used in applications are those that retrieve information from one or more tables of the database [8]. The select clause determines which fields (columns) constitute the query output, the from clause determines which tables are used and the join determines the criterion for joining rows from different tables. Then the where clause filters the rows based on some other criteria. The group by clause indicates how to combine the selected rows and the having clause performs a final filter based on other conditions. Additionally, the order by clause determines how to order the resulting set of data.

When testing SQL queries we must take into account a number of issues that are specific to this language, such as the non procedural character of SQL, the high input and output spaces, the dependence on the database schema, the presence of unknown values (due to the use of tri-valued logics) and in general, the existence of few specifically tailored adequacy criteria to assess the test cases [9].

Mutation testing has been demonstrated as a powerful approach to evaluate test cases and for comparing different testing strategies or techniques. Empirical studies show that the generated mutants provide a good indication of the fault detection ability of a test suite [1,2]. Some previous works in database testing have used mutants to evaluate the fault detection capability of database test cases $[4,5,11]$ in order to assess the effectiveness of test generation techniques. In [3] a set of SQL mutants based on features present in a conceptual model of the database schema is presented. However, all the above approaches are either manual or focus on a reduced subset of SQL features.

In [10] we described a set of mutation operators for SQL select queries that covers a wide range of the SQL syntax and semantics. The goal of this paper is to present some internal details about the tool that automates the mutation process. This tool is named SQLMutation and it is publicly accessible at http://in2test.lsi.uniovi.es/sqlmutation. It allows the mutants to be generated interactively from a Web browser, or from other programs by consuming a Web service.

In the rest of the paper we provide an overview of the mutants (Section 2), some technical details about the tool (Section 3) and typical faults (Section 4). Finally, we present some conclusions (Section 5).

\section{SQL mutants}

The mutation operators are organized into four categories:

- $S C$ - SQL clause mutation operators: These perform mutations on the main clauses: select, join, sub-queries, group by, union, order by and aggregate functions.

- OR - Operator replacement mutation operators: These are similar to the expression modification operators described in [7] plus additional operators specific to between and like predicates. 


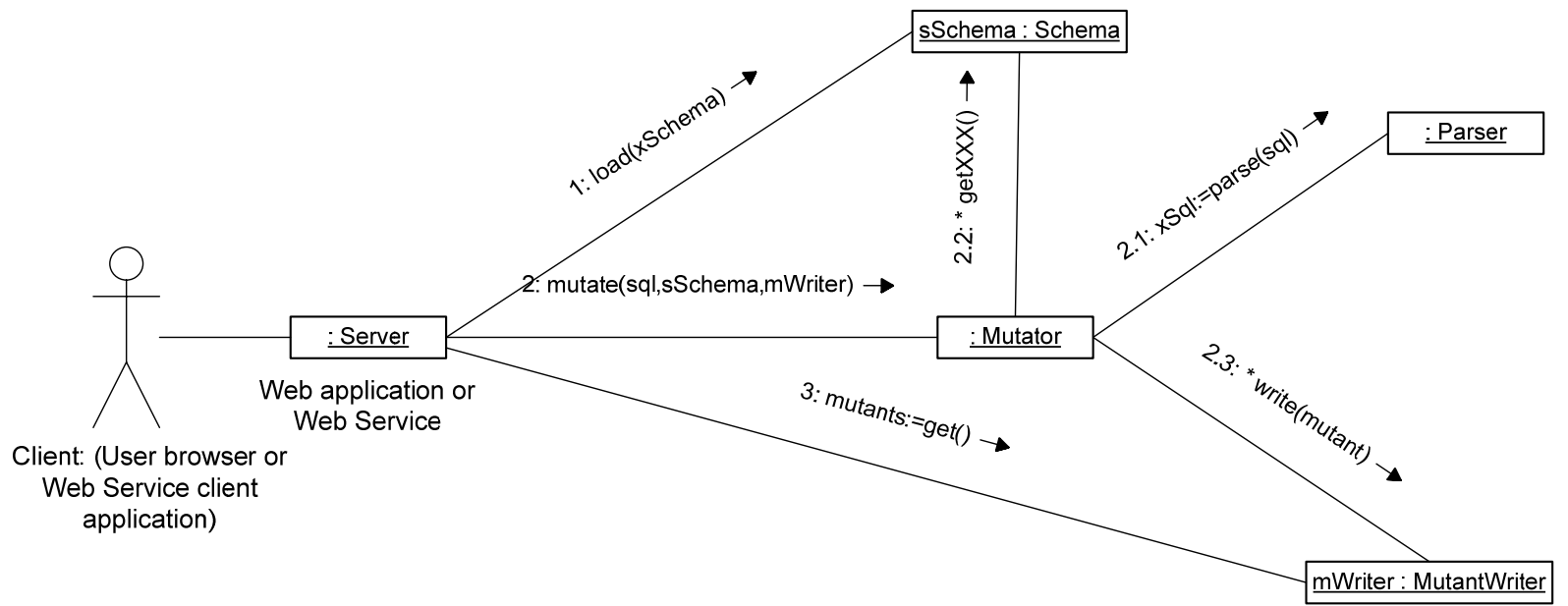

Figure 1. Architecture of the tool

- $\quad$ NL - NULL mutation operators: Mutations related to the handling of null values, whose aim is to ensure that test cases exist that exercise the nulls both in the conditions and the query outputs.

- IR - Identifier replacement mutation operators: Replacement of columns, constants and query parameters that are present either in the query or in the tables used by the query.

Each category defines several mutation operators or mutant types. As most of the operators can be applied in different SQL clauses, each type is further decomposed into subtypes, each of which refers to a particular mutant type when applied to a given clause. The mutation operators are described in detail in [10].

\section{The SQLMutation tool}

Figure 1 depicts the main architecture of the tool. Two implementations of the Server front-end are available: a Web application for interactive usage and a Web service for use from other applications. The core of the system is the Mutator that creates the mutants. This is helped by Schema (which provides information about all elements in the database schema), the Parser (transforms the SQL query into an internal representation) and the MutantWriter which stores the mutants that are being generated and returns them to the Server in a suitable format.

In the following subsections each of the components is described in more detail.

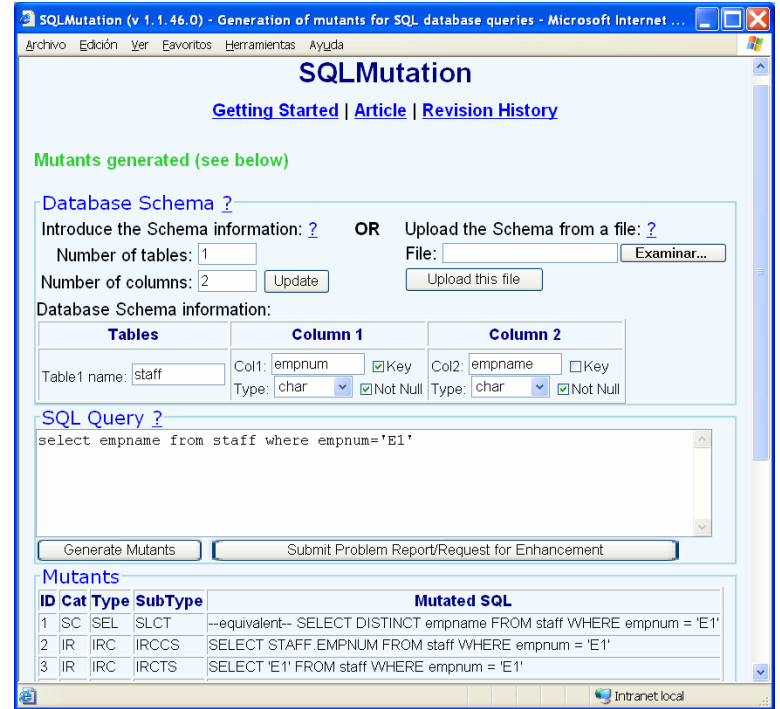

Figure 2. Main Screen (Web interface)

\subsection{Server front-ends}

The mutation process requires the user to specify both the SQL query and information about the database schema (table names, column names, data types as well as primary keys and null constraints). The first frontend to SQLMutation is a Web application that allows the user to introduce this information from a browser and then generate the mutants. Figure 2 shows a sample of the main screen.

The mutants are presented to the browser in a table, including the classification of each mutant (category, type and subtype) along with the mutated SQL. An 
additional form is available to submit problem reports or requests for enhancement.

The second front-end is a Web service that provides a method getMutants which takes two input parameters: the SQL query to be mutated and the database schema. The database schema must be supplied in an internal XML format used by SQLMutation. The following is a sample of a schema that declares a table with two columns, the first is the primary key, and the second has a not null constraint:

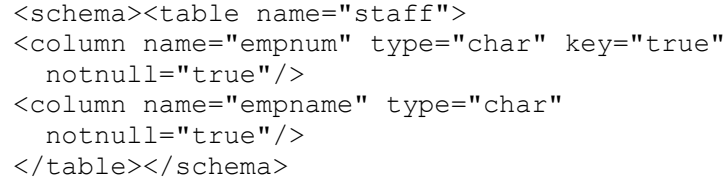

The Web service response is an XML formatted string which contains all the mutants and/or error information if applicable. A sample of this response is presented below:

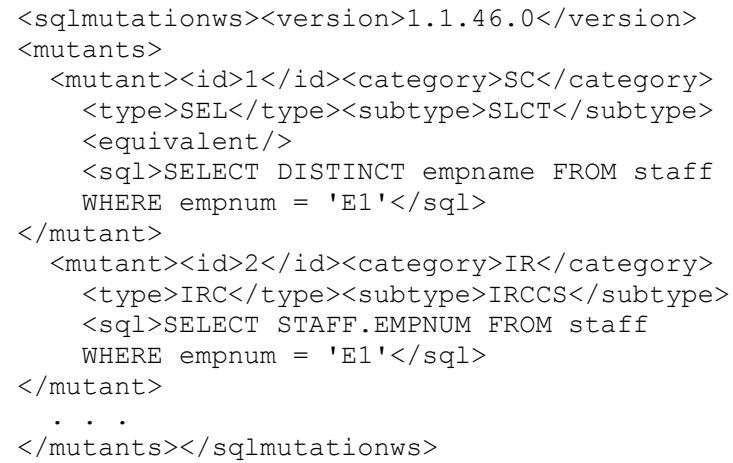

Using the Web service front-end, third party applications written in different platforms such as Java or .NET are able to integrate the SQLmutation tool. In the online documentation, two sample clients written in VB.NET and Java with Eclipse Web Tools Platform are supplied.

\subsection{Schema, Parser and MutantWriter}

Before generating the mutants, the Schema class is instantiated into the sSchema object and loaded with the XML representation of the database schema (see above). During the mutation process the sSchema object is called repeatedly to obtain columns, constants, their data types, constraints and to resolve the table aliases.

The Parser transforms the SQL query into an internal XML format by replacing the keywords by elements and then reorganizing the resulting XML document in order to produce a suitable structure of the query. Keywords are represented as elements and columns, tables, parameters and constants as text. An example of the internal XML representation of the query presented in Figure 1 is the following:

$<$ sql $><$ select $>$ empname $</$ select $>$

$<$ from $>$ staff $</$ from $>$

$<$ where $>$ empnum<eq/> E1 '</where $></$ sql $>$

While the Mutator generates each mutant it calls the MutantWriter interface which stores an applicationdependent representation of each mutant. For instance, the implementation of the interface used in the Web application stores the mutants in an HTML table object, and the Web service stores the mutants in XML.

\subsection{Mutator}

The core of SQLMutation is a set of classes (represented by the Mutator in Figure 1) that receives the schema and the SQL query, calls the Parser and loads the XML representation of the query into a DOM model. Then the Mutator traverses recursively each element in the DOM and whenever it finds an element or text node performs one or more of the following operations:

- Scope setting: Column references must be mapped to the corresponding table in the database schema that declares it. When visiting each select clause (a query may have more than one select clause when there is a union or a subquery) or each join clause, a list of the tables that are in the scope of this node is updated. An additional list is created including the columns, constants and parameters needed for the replacements made in by the IR category of mutants.

- Column resolution: When a text node is found, the Schema determines whether it is a constant, parameter or column reference. In the last case, the table that declares the column is searched for in the list. When a table appears more than once, the references are mapped to the corresponding aliased table.

- Mutation: If the visited node is an element or text node suitable for generating one or more mutants, a clone of it is first created and then every necessary transformation to obtain the mutant(s) is performed on the clone. Immediately after generating each mutant, the MutantWriter is called to save the transformed clone into a new mutant.

After finishing the mutation process, the server application will call the MutantWriter to get all generated mutants and send them back to the client. 


\section{Using the SQL mutants}

Next, we will present some results of the faults introduced in a set of queries developed by seven students during an exercise of SQL development. The exercise consisted in writing four queries using the same database schema. All of the queries must join three tables using different kinds of join-types. The queries have simple where-conditions and two of them have a group by and having clauses.

We generated the mutants of the desired queries, removed the equivalent mutants and executed them against the test databases developed by the students. The mutation scores are presented in Table 1.

Table 1. Mutation Scores

\begin{tabular}{|c|c|c|c|c|c|}
\hline Category & Q1 & Q2 & Q3 & Q4 & Tot. \\
\hline IR - Identifier Replac. & 98,8 & 94,2 & 87,9 & 78,1 & 89,1 \\
\hline $\mathrm{NL}-$ Nulls & & 96,4 & 77,1 & 60,0 & 78,4 \\
\hline OR - Operator Replac. & 87,4 & 80,0 & 81,4 & 74,0 & 80,3 \\
\hline SC - SQL Clauses & 86,8 & 39,0 & 50,4 & 69,6 & 59,6 \\
\hline Tots & 89,6 & 80,7 & 75,0 & 73,6 & 78,5 \\
\hline
\end{tabular}

We examined the queries written after the exercise and counted the kind of faults committed in each one. The result is presented in Table 2 .

Table 2. Faults committed in the queries

\begin{tabular}{|l|c|c|c|c|c|}
\hline & Q1 & Q2 & Q3 & Q4 & Tot. \\
\hline Omitted distinct in select & 1 & & & & 1 \\
\hline One join with wrong type & & & & 3 & 3 \\
\hline Two joins with wrong type & 3 & 3 & & & 6 \\
\hline Use a wrong SQL86 join & & 1 & & 1 & 2 \\
\hline Join incorrect tables & & 1 & & & 1 \\
\hline Wrong columns in order by & & 1 & & & 1 \\
\hline Order by omitted & & 3 & 4 & 4 & 11 \\
\hline Unnecessary IS NULL & & 1 & & & 1 \\
\hline Omitted IS NULL & & & 5 & 4 & 11 \\
\hline Wrong columns in select-list & & 1 & & & 1 \\
\hline
\end{tabular}

The faults were related to the distinct quantifier, the join and order by clauses, the use of the is null predicate and the usage of wrong columns. All of them are faults that are represented by the mutants. Most of the faults committed are represented by the mutants that belong to the SC and NL categories, which are those that have achieved the lowest mutation scores (Table 1).

The above provides us with some insight into how well the mutants model the real faults committed in this exercise. However, if we use the SQL mutants to assess the adequacy of database tests developed while writing a query, some relevant faults may not be represented by the mutants. For instance, a frequent fault is the omission of the order by clause. If we generate the mutants of a faulty query that has an incorrect order by clause, the mutants can assist us in detecting this fault. But, if the faulty query does not include an order by clause, no mutants will be generated for this clause and consequently this fault will not be detected. A potential new mutation operator may be needed in that case to insert additional order by clauses.

\section{Conclusions}

We presented a tool that automatically generates mutants of SQL database queries. The tool can be used interactively or integrated in other tools that consume the Web service exposed. This system is intended to be used by researchers in database applications testing for assessing the adequacy of database test cases and for comparing different techniques.

The set of the mutants generated covers a wide range of SQL features, although there is room for improvement and refinement of the mutants. We encourage researchers to use it and thus, to collaborate in its evolution.

\section{Acknowledgements}

This work was funded by the Department of Education and Science (Spain) and FEDER funds, under the National Program for Research, Development and Innovation, Projects IN2TEST (TIN2004-06689-C03-02) and REPRIS (TIN200524792-E).

\section{References}

[1] J. Andrews, L. Briand, Y. Labiche, Is Mutation an Appropriate Tool for Testing Experiments?, Proc. of the 27th International Conference on Software Engineering. ACM Press, New York, NY, USA, 2005, pp. 402-411.

[2] J. Andrews, L. Briand, Y. Labiche, A. S. Namin, Using Mutation Analysis for Assessing and Comparing Testing Coverage Criteria, IEEE Transactions on Software Engineering 32(8) 608-624.

[3] W.K. Chan, S.C. Cheung, T.H. Tse, Fault-Based Testing of Database Application Programs with Conceptual Data Model, Proc. of the fifth International Conference on Quality Software, IEEE Computer Society Press, Los Alamitos, California, 2005, pp. 187-196. 
[4] Y. Deng, P. Frankl, D. Chays, Testing Database Transactions with AGENDA. Proc. of the 27th International Conference on Software Engineering, ACM Press, New York, NY, USA, 2005, pp. 78-87.

[5] S. Elbaum, G. Rothermel, S. Karre, M. Fisher II, Leveraging User-Session Data to Support Web Application Testing, IEEE Transactions on Software Engineering 31(3) (2005) 187-202.

[6] International Standards Organisation, Information technology - Database languages - SQL, ISO/IEC 9075:1992, third edition.

[7] K.N. King, A.J. Offutt. A Fortran Language System for Mutation-Based Software Testing. Software Practice and Experience 21(7) (1991) 686-718.

[8] R. Pönighaus, 'Favourite' SQL-Statements - An Empirical Analysis of SQL-Usage in Commercial Applications. Proc. of the 6th International Conference on Information Systems and Management of Data (LNCS, vol. 1006), Springer, 1995, pp. 75-91.

[9] J. Tuya, M.J. Suárez-Cabal, C. de la Riva, A practical guide to SQL white-box testing, ACM SIGPLAN Notices, 41(4) 36-41, 2006.

[10] J. Tuya, M.J. Suárez-Cabal, C. de la Riva, Mutating Database Queries, Information and Software Technology, 2006. (In press, doi:10.1016/j.infsof.2006.06.009).

[11] W.T. Tsai, D. Volovik, T.F. Keefe, Automated test case generation for programs specified by relational algebra queries, IEEE Transactions on Software Engineering 16(3) (1990) 316-324. 